\title{
Hexagonal Phase of Polyethylene Fibers under High Pressure
}

\author{
Shinsuke Tsubakihara, Atsushi Nakamura, ${ }^{\dagger}$ and Munehisa Yasuniwa \\ Department of Applied Physics, Faculty of Science, Fukuoka University, \\ Jonan-ku, Fukuoka 814-01, Japan
}

(Received January 21, 1991)

\begin{abstract}
The hexagonal phase of a constrained ultra-drawn polyethylene (PE) fiber (UDPF) was studied in detail. The melting process of the constrained UDPF was examined at atmospheric pressure by DSC and X-ray measurement. The hexagonal phase appears prior to melting in the constrained UDPF. The phase diagram showing the pressure change of the hexagonal phase was determined by high pressure DTA up to about $600 \mathrm{MPa}$. The temperature range of the hexagonal phase increases with pressure and coincides with that of so-called "high pressure phase" (HPP) of it above about $300 \mathrm{MPa}$. That is, the hexagonal phase of the constrained UDPF is the same as HPP. The phase diagrams of three kinds of PE samples, two constrained UDPFs and a bulk sample composed of extended-chain crystals, determined by high pressure DTA, were compared with one another. The constraining force applied to these PE samples considerably raises the melting temperature of HPP, while it has almost no effect on the transition temperature from orthorhombic phase to HPP. By constraining force, HPP of UDPF appears even in the low pressure region that includes atmospheric pressure.

KEY WORDS Polyethylene / Ultra-Drawn Fiber / Ultra-High Molecular

Weight / High Pressure / DSC / DTA / X-Ray / Hexagonal Phase / High

Pressure Phase / Extended-Chain Crystal/
\end{abstract}

About ten years ago, high-modulus and high-strength polyethylene (PE) fibers were produced by ultra-drawing techniques from ultra-high molecular weight (UHMW) PE gels. ${ }^{1-3}$ Recently they have been widely applied to textile and composite materials. The melting processes of these fibers have been studied in detail because their melting temperatures make a limit on the application of them at elevated temperature. Pennings et al. $^{4}$ and Lemstra et al. ${ }^{5}$ investigated the melting process of constrained ultra-drawn PE fibers (UDPF) by $\mathrm{X}$-ray measurement and showed that a hexagonal phase appears prior to melting in the heating process.

Yasuniwa et al. ${ }^{6}$ and Bassett et al. ${ }^{7}$ found "high pressure phase" (HPP) for a bulk sample of PE under high pressure above about
$300 \mathrm{MPa}$. The crystal structure of HPP was clarified to be hexagonal by X-ray measurement. ${ }^{8}$ That is, HPP is another hexagonal phase of PE. Then the precise temperature change of lattice parameters in orthorhombic phase and HPP were obtained. ${ }^{9}$ Takemura et al. ${ }^{10,11}$ studied the physical properties of HPP by various experimental methods and characterized HPP to be like a liquid crystal. Yamamoto et al. ${ }^{12}$ showed by X-ray measurement that the conformational disordering of molecular chains occurs in HPP.

It is interesting to study the relation between the hexagonal phase of constrained UDPF and HPP. However, the pressure change of the hexagonal phase of the constrained UDPF has not yet been studied due to experimental difficulties. In this work, the detailed melting

† Present address: Laboratory of Fluoroplastics, Nippon Valqua Industries, Ltd., Tanasawa, Atsugi-shi, Kanagawa 243-02, Japan. 
process of the constrained UDPF was examined under high pressure, and its phase diagram that shows the pressure change of its hexagonal phase was determined. The relation between the hexagonal phase and HPP and formation mechanism of the hexagonal phase are discussed.

\section{EXPERIMENTAL}

UDPFs used in this work were "Spectra 900" (Allied Signal Co., viscosity average molecular weight, $M_{v}=c a . \quad 2 \times 10^{6}$ ) and "Tekmilon NA310" (Mitsui Petrochemical Industries Co., $M_{v}=c a .7 \times 10^{5}$ ) made from UHMW PE. In this paper, they are denoted by UDPF-1 and UDPF-2, respectively. A bulk sample prepared by high pressure crystallization of an unfractionated high density PE $\left(M_{v}=2.2 \times 10^{5}\right)$ supplied by Showa Denko Co. was also used.

The melting processes of PE samples were examined using a DSC apparatus (Rigaku Denki low temperature thermal analyzer) at atmospheric pressure. Five types of samples (samples A-E) shown in Table I were prepared from UDPF-1. Samples A and B were cut to a length of $20 \mathrm{~mm}$ from the original fiber and were wound around polytetrafluoroethylene (PTFE) rod tightly and loosely, respectively. Samples C ( $5 \mathrm{~mm}$ in length) and $\mathrm{D}$ (1 $\mathrm{mm}$ in length) were shrinkage-free fibers cut off from the same original fiber. The difficulty of the thermal shrinkage of UDPF increases with length and strength of constraining force. Accordingly, the difficulty of the thermal shrinkage of fiber samples A-D decreases from A to D. On the other hand, bulk sample $\mathrm{E}$ was prepared from the original fiber as follows. The fiber was maintained at $160^{\circ} \mathrm{C}$ in an atmosphere of nitrogen and melted completely. The melted PE was then cooled slowly and crystallized to a bulk sample. All samples were put into aluminium containers, and DSC curves were obtained at a heating rate of $4 \mathrm{~K} \mathrm{~min}^{-1}$.

The structural change of UDPF-1 at
Table I. Samples for DSC measurement

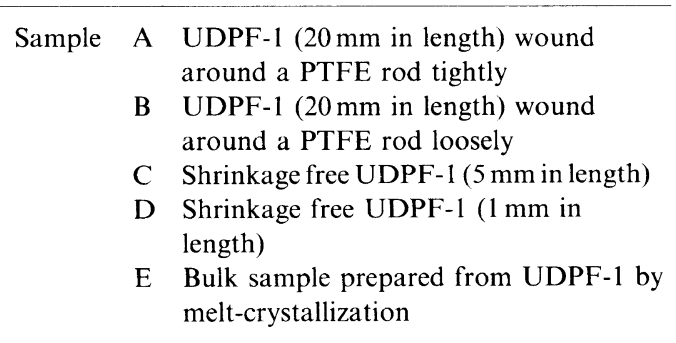

(a)

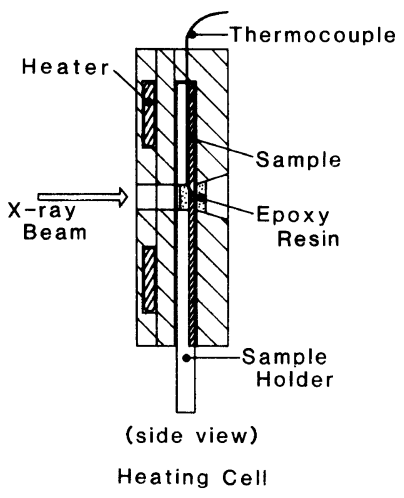

(b)

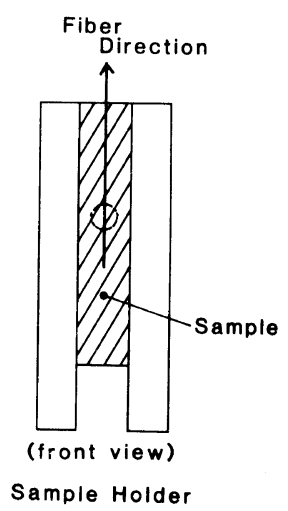

Figure 1. Schematic pictures of (a) heating cell for X-ray measurement and (b) sample holder.

atmospheric pressure was examined by wideangle X-ray measurement in the heating process. Figure 1 shows a heating cell and a sample holder made of brass. The fiber sample was fixed on the sample holder and coated with epoxy resin in order to prevent its thermal shrinkage. The temperature of the sample was measured by an alumel-chromel thermocouple 
located at a distance, $1 \mathrm{~mm}$, from the center of an aperture for X-ray exposure. A Rigaku Rotaflex RU-200 (60 kV, $200 \mathrm{~mA}$ ) was used as a X-ray generator. Mo-K $\alpha \mathrm{X}$-ray beam filtered with zirconium was applied to the sample perpendicular to the fiber direction.

X-Ray fiber patterns were obtained with a flat-film camera. Equatorial diffraction patterns were also recorded using a diffractometer. The diffractometer was scanned over a range of $8.5^{\circ}<2 \theta<12^{\circ}$. Between these angles, (110) and (200) reflections of the orthorhombic phase and (100) reflection of the hexagonal phase were observed.

High pressure DTA of constrained UDPFs and bulk samples was carried out up to $600 \mathrm{MPa}$ using the apparatus described elsewhere. ${ }^{13}$ As a pressure transmitting fluid, a low viscosity silicone oil (Shin-Etsu Chem. Co.) was used. Hydrostatic pressure was measured within $\pm 1 \mathrm{MPa}$ by a Bourdon's gauge (Heise Co.) connected to the high pressure vessel. Heating rate was precisely controlled by an automatic temperature controller. Fiber samples were wound around the junction of an alumel-chromel thermocouple tightly and coated with epoxy resin in order to prevent their thermal shrinkage. Bulk samples were stuck on the junction and covered by epoxy resin likewise. DTA curves were obtained at a heating rate of $5 \mathrm{~K} \mathrm{~min}^{-1}$.

\section{RESULTS AND DISCUSSION}

Figure 2 shows the DSC curves for five types of samples shown in Table I. Bulk sample E shows a single endothermic peak $\left(\mathrm{ca} .131^{\circ} \mathrm{C}\right)$ that corresponds to the melting of folded-chain crystals (FCC). On the other hand, the DSC curves of fiber samples A-D show multiple peaks, and their temperatures are higher than that of bulk sample E. On the DSC curves of samples $A-C$, three endothermic peaks denoted by 1, 2, and 3 appear up to complete melting. Peak 1 at about $145^{\circ} \mathrm{C}$ is attributed to the melting of unconstrained ECCs included

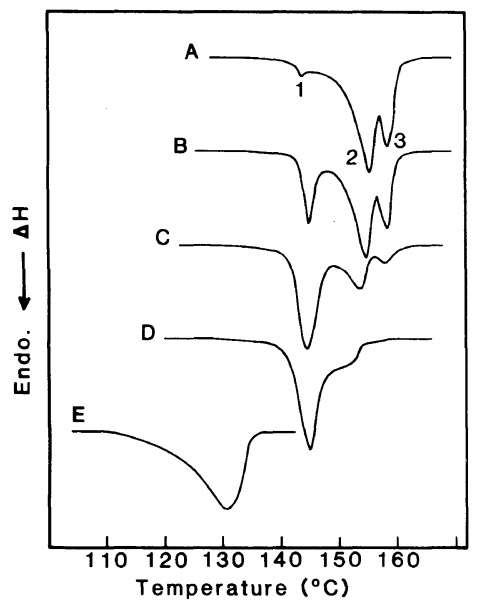

Figure 2. DSC curves of five types of samples in Table I (heating rate, $4 \mathrm{~K} \mathrm{~min}^{-1}$ ). The alphabetical symbols of the DSC curves correspond to those of the samples in Table I.

in the fiber samples. ${ }^{5}$ The latent heat of peak 1 increases and those of peaks 2 and 3 decrease from $A$ to $D$. In the case of sample A constrained most tightly, peak $1\left(144^{\circ} \mathrm{C}\right)$ has considerably small latent heat, and the major part of the sample melts through peaks 2 $\left(155^{\circ} \mathrm{C}\right)$ and $3\left(159^{\circ} \mathrm{C}\right)$. On the other hand, the major part of sample $\mathrm{D}$, which was constrained most loosely, melts at peak 1 , and distinct peaks 2 and 3 do not appear. These results indicate that tightly constrained UDPF melts through peaks 2 and 3 instead of peak 1 . Lemstra ${ }^{5}$ attributed peaks 2 and 3 to the transition from the orthorhombic phase to the hexagonal one (ortho.-hexa. transition) and the melting of the hexagonal phase, respectively. To clarify how the structure of constrained UDPF changes at the temperatures of peaks 2 and 3, X-ray measurement was carried out in the heating process.

Figure 3 shows the X-ray fiber patterns of constrained UDPF-1, taken using the apparatus shown in Figure 1 and a flat-film camera. Figure 3a was taken at room temperature, and Figure $3 \mathrm{~b}$ was taken at $156^{\circ} \mathrm{C}$ between the temperatures of peaks 2 and 3 in Figure 2. The diffuse scattering rings of both photographs are 
(a)

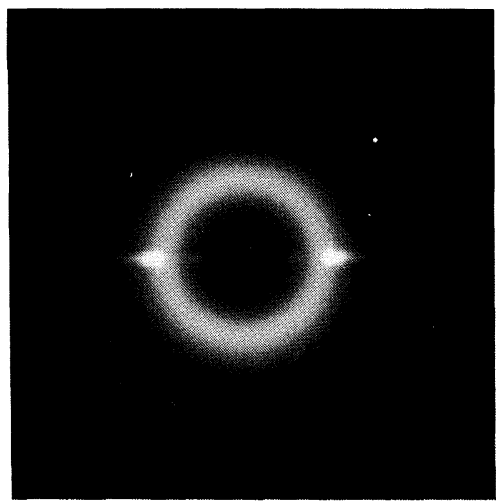

(b)

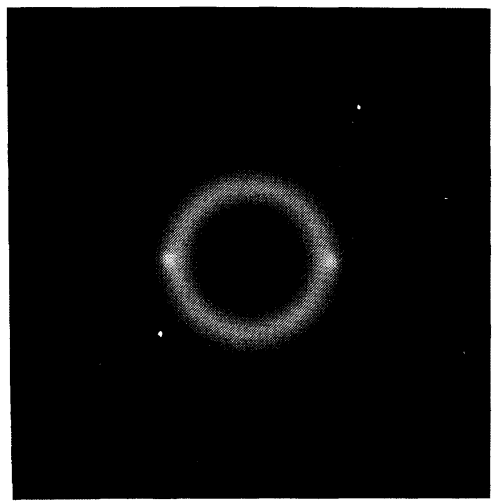

Figure 3. X-Ray fiber patterns of constrained UDPF-1: (a) orthorhombic structure (room temperature); (b) pseudo-hexagonal structure $\left(156^{\circ} \mathrm{C}\right)$. Both photographs were obtained using the apparatus shown in Figure 1 and a flat-film camera. Fiber axis is vertical.

the amorphous halo of the epoxy resin used to coat the sample surface. The fiber pattern obtained at room temperature (Figure $3 a$ ) is orthorhombic. On the other hand, the fiber pattern in Figure $3 b$ is hexagonal because any Bragg reflections are not observed except for two strong equatorial ones. From this fiber pattern, it is shown that the constrained UDPF has the pseudo-hexagonal structure that does not have periodic order in the direction of molecular chains between the temperatures of peaks 2 and 3.

Figure 4 shows the precise temperature change of the X-ray equatorial diffraction pattern of constrained UDPF-1 in the heating process. At $155^{\circ} \mathrm{C}$ and above it, (110) and (200)

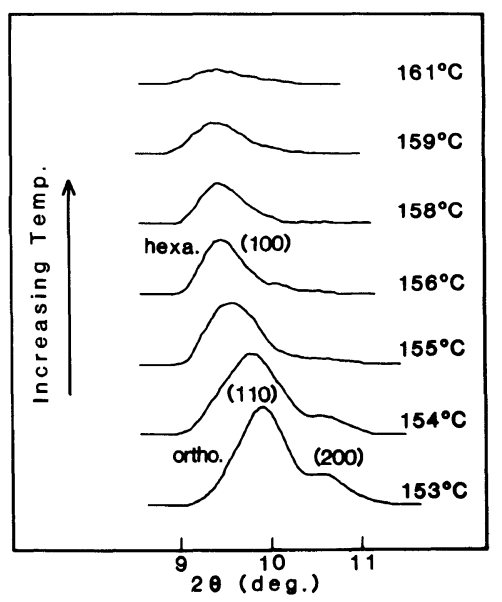

Figure 4. Temperature change of the X-ray equatorial diffraction pattern of constrained UDPF-1.

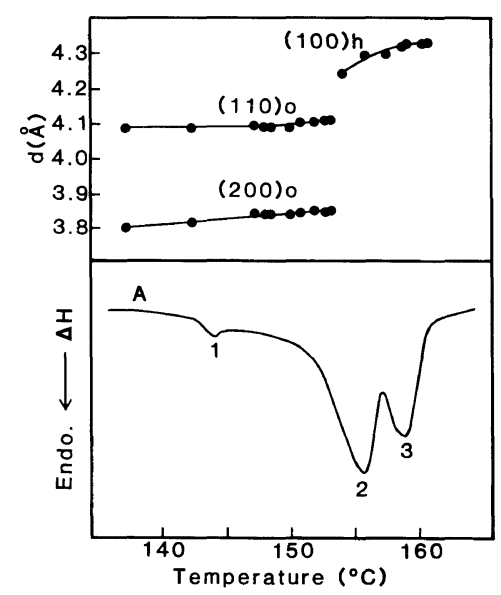

Figure 5. Temperature dependence of lattice spacing calculated from the X-ray diffraction pattern of constrained UDPF-1 and the DSC curve of sample A in Figure 2. Subscripts $o$ and $h$ denote the reflections of the orthorhombic and hexagonal phases, respectively.

reflections of the orthorhombic phase disappear, and (100) reflection of the hexagonal phase appears. The diffraction intensity of (100) reflection decreases gradually with temperature and disappears at $161^{\circ} \mathrm{C}$ and the halo of liquid is left. These results indicate that the ortho.-hexa. transition occurs at $155^{\circ} \mathrm{C}$ and hexagonal crystals melt completely at $161^{\circ} \mathrm{C}$.

Figure 5 shows the temperature dependence 


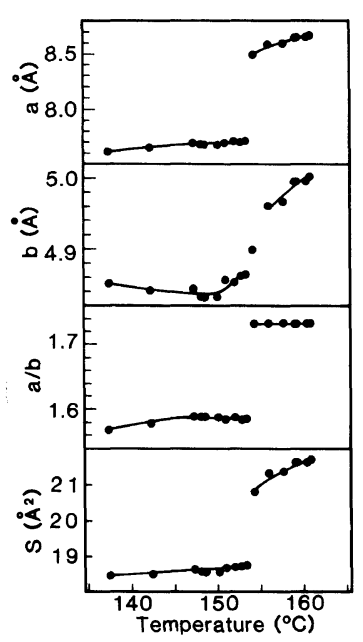

Figure 6. Temperature changes of lattice parameters $a$, $b$, the ratio $a / b$, and $S . S$ is the cross section of one molecule perpendicular to the chain axis calculated from $S=(1 / 2) a b$.

of lattice spacing calculated from the X-ray diffraction pattern of constrained UDPF- 1 and the DSC curve of sample A (shown in Figure 2) on the same temperature scale. Within the temperature range of peak 2, (110) and (200) reflections of the orthorhombic phase disappear, and (100) reflection of the hexagonal phase appears. From this result, peak 2 of constrained UDPF is attributed to the ortho.-hexa. transition. Spacing of (100) lattice planes in the hexagonal phase increases with temperature. Since (100) reflection disappears completely at the end of peak 3 , this peak corresponds to the melting of the hexagonal phase.

Figure 6 shows the temperature change of the lattice parameters of constrained UDPF-1. For the hexagonal phase, orthohexagonal representations given by

$$
\begin{aligned}
& a(\text { orthohexagonal })=2 a(\text { hexagonal }) \cos 30^{\circ} \\
& b(\text { orthohexagonal })=2 a(\text { hexagonal }) \sin 30^{\circ}
\end{aligned}
$$

are used. $S$ is the cross section of one molecular chain perpendicular to the chain axis. The $a$ axis in the orthorhombic phase expands

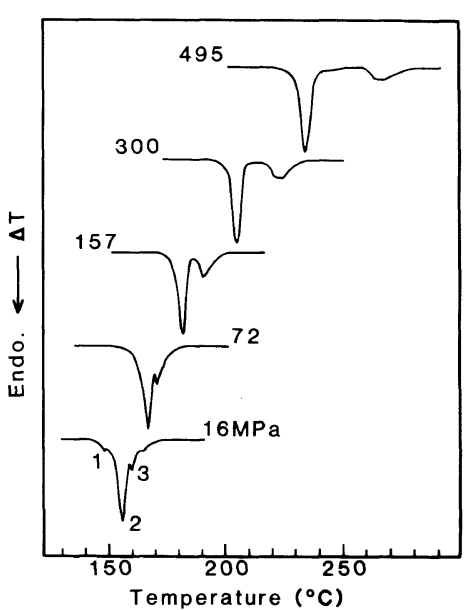

Figure 7. DTA curves of constrained UDPF-1 at various pressures (heating rate, $5 \mathrm{~K} \mathrm{~min}^{-1}$ ).

gradually with temperature, while the $b$ axis in the phase shrinks only slightly. At the ortho.-hexa. transition temperature, lattice parameters $a, b, a / b$ ratio, and $S$ increase discontinuously, and the $a$ and $b$ axes of the hexagonal phase continue to expand up to complete melting. These changes of the lattice parameters are similar to those in the case of the transition from the orthorhombic phase to HPP in a bulk sample under high pressure. ${ }^{9}$ From this fact, it is suggested that both ortho--hexa. transition in the constrained UDPF and transition to HPP in the bulk sample occur with the same structural change.

Figure 7 shows the DTA curves of constrained UDPF-1 at various pressures. The peak profile at the lowest pressure, $16 \mathrm{MPa}$, is different slightly from that of the DSC curve of sample A in Figure 2, due to the difference between their constraining modes. The temperature difference between peaks 2 and 3 , that is, the temperature range of the hexagonal phase increases with pressure. The peak profile at $495 \mathrm{MPa}$ is similar to that of a bulk sample at the same pressure, while the temperature difference between two peaks is larger than that of the bulk sample. ${ }^{6}$ Peaks 2 and 3 of the bulk sample, which appear above about $300 \mathrm{MPa}$, 


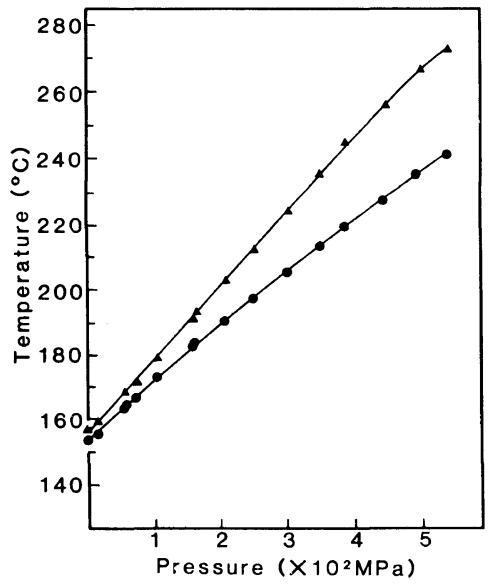

Figure 8. Phase diagram of constrained UDPF-1: ortho.-hexa. transition temperature (peak 2 in Figure 7); $\boldsymbol{\Delta}$, melting temperature of the hexagonal phase (peak 3 in Figure 7). These temperatures were obtained from the peak temperatures of DTA curves.

are attributed to the transition from the orthorhombic phase to HPP and the melting of HPP, respectively.

The phase diagram of constrained UDPF-1, determined by DTA measurement, is shown in Figure 8. The marks and $\boldsymbol{\Delta}$ are the ortho--hexa. transition temperature and the melting temperature of the hexagonal phase, respectively. These temperatures were obtained from the peak temperatures of DTA curves. The temperature range of the hexagonal phase increases with pressure and coincides with that of HPP of the constrained UDPF under high pressure above about $300 \mathrm{MPa}$. That is, the hexagonal phase of constrained UDPF is the same as HPP. Hereafter the term HPP is used for the hexagonal phase of constrained UDPF.

Figure 9a shows the DTA curves of bulk sample E shown in Table I, prepared from UDPF-1 by melt-crystallization at atmospheric pressure. Below about $300 \mathrm{MPa}$, there is an only endothermic peak that corresponds to the melting of FCCs (orthorhombic phase). On the other hand, two endothermic peaks attributed to the transition from the orthorhombic phase (a)

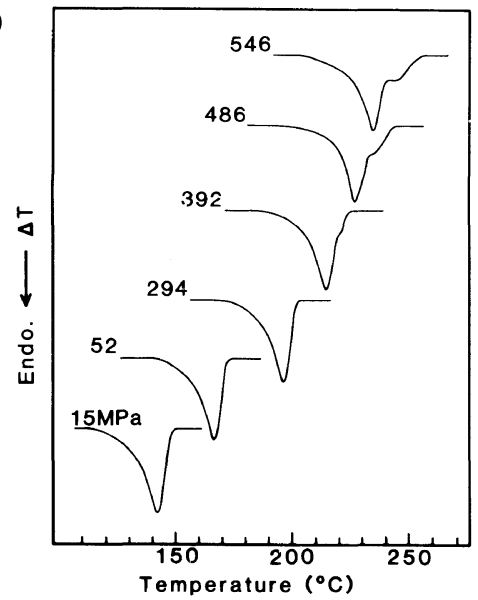

(b)

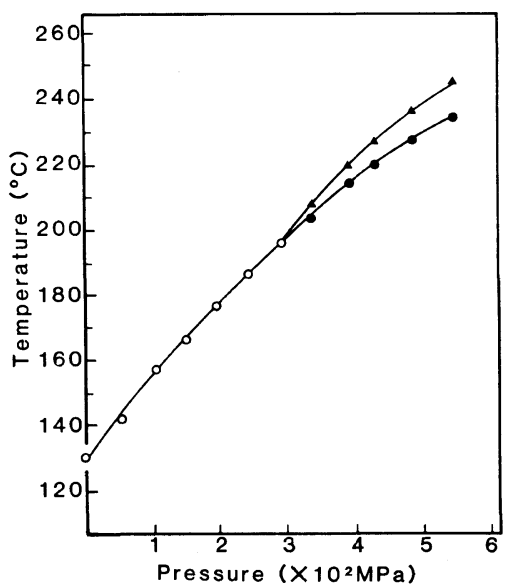

Figure 9. DTA curves (a) and phase diagram (b) of bulk sample $\mathrm{E}$ in Table $\mathrm{I}$ (heating rate, $5 \mathrm{~K} \mathrm{~min}^{-1}$ ): $\bigcirc$, melting temperature of the orthorhombic phase; $\mathbf{O}$, transition temperature from the orthorhombic phase to HPP; melting temperature of HPP. These temperatures were obtained from the peak temperatures of DTA curves.

to HPP and the melting of HPP appear above about $300 \mathrm{MPa}$. The phase diagram of bulk sample $\mathrm{E}$ is shown in Figure $9 \mathrm{~b}$. The mark $\bigcirc$ is the melting temperature of the orthorhombic phase. The marks and $\boldsymbol{\Delta}$ are the transition temperature from the orthorhombic phase to HPP and the melting temperature of HPP, respectively. As shown in the figure, HPP of the UHMW-PE bulk sample exists only above about $300 \mathrm{MPa}$. 


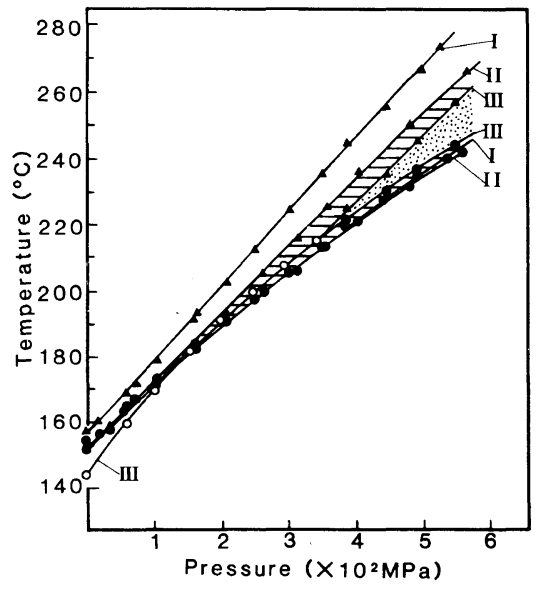

Figure 10. Phase diagrams of constrained UDPF-1 denoted by I, constrained UDPF-2 denoted by II, and bulk sample III $\left(M_{v}=2.2 \times 10^{5}\right): \bigcirc$, melting temperature of the orthorhombic phase; $\mathbf{O}$, transition temperature from the orthorhombic phase to HPP (hexagonal phase); $\boldsymbol{\Delta}$, melting temperature of HPP. All phase diagrams were determined by high pressure DTA (heating rate, $5 \mathrm{~K} \mathrm{~min}^{-1}$ ), and the transition and melting temperatures were obtained from the peak temperatures of DTA curves.

Though bulk sample E was prepared from UDPF-1, the phase diagrams of constrained UDPF-1 (Figure 8) and bulk sample E (Figure $9 b)$ are considerably different from each other. The temperature range of HPP of the constrained UDPF is broader than that of bulk sample E, and HPP of the constrained fiber appears even at atmospheric pressure. It was reported that chemical crosslinks of PE, introduced by $\gamma$-irradiation, stabilize HPP even at atmospheric pressure because the chemical crosslinks confine the conformational disorder of molecular chains above the melting temperature of the orthorhombic phase. ${ }^{14,15}$ Constraining the thermal shrinkage of UDPF also confines the conformational disordering of molecular chains in the heating process. Consequently, the temperature range of HPP extends, and the lowest pressure limit decreases to atmospheric pressure.

Figure 10 shows the phase diagrams of three kinds of PE samples, constrained UDPF-1, UDPF-2, and a bulk sample denoted by I, II, and III, respectively. The phase diagrams of UDPF-2 (II) and the bulk sample (III) were also determined by high pressure DTA. The bulk sample was prepared from the unfractionated high density PE $\left(M_{v}=2.2 \times 10^{5}\right)$ by high pressure crystallization at $500 \mathrm{MPa}$. From electron microscopic observation, it was previously confirmed that the bulk sample is composed of large ECCs of the order of $\mu \mathrm{m}$ in thickness. ${ }^{16}$ Therefore, all samples consist of ECCs. However, the constraining force applied to the samples differs in each case. UDPF-1 and UDPF-2 were constrained tightly by winding the fibers on the thermocouple junction, while the bulk sample was not constrained by particular method. UDPF-1 was constrained more tightly than UDPF-2 because UDPF-1 made of higher molecular weight PE $\left(M_{v}=c a .2 \times 10^{6}\right)$ may have more entanglements than UDPF-2 made of lower molecular weight PE $\left(M_{v}=c a .7 \times 10^{5}\right)$. The entanglements constrain molecular conformational disordering in the heating process. The strength of constraining force decreases from I to III in the figure.

As shown in Figure 10, the temperature ranges of HPPs of three kinds of samples are considerably different from one another. The melting temperature of HPP is raised remarkably from III to I by the constraining force. UDPF-1 (I) constrained most tightly has the highest melting temperature of HPP among three kinds of samples, while the bulk sample (III), i.e., the most loosely constrained sample has the lowest melting temperature of HPP. On the other hand, their transition temperatures from the orthorhombic phase to HPP are very close to one another. This indicates that the transition temperature does not vary much by constraining force. Below about $300 \mathrm{MPa}$, the melting temperature of HPP raised by the constraining force for UDPF becomes higher than the transition temperature which does not change by the constraining force. Consequently, HPP, the hexagonal phase of the constrained UDPF, appears even in the low 
pressure region that includes atmospheric pressure.

Acknowledgment. The authors thank Mr. K. Yagi of Mitsui Petrochemical Industries Co. for providing PE fibers.

\section{REFERENCES}

1. P. Smith and P. J. Lemstra, J. Mater. Sci., 15, 505 (1980).

2. P. Smith and P. J. Lemstra, Polymer, 21, 1341 (1980).

3. P. Smith, P. J. Lemstra, and H. C. Booij, J. Polym. Sci., Polym. Phys. Ed., 19, 877 (1981).

4. A. J. Pennings and A. Zwijnenburg, J. Polym. Sci., Polym. Phys. Ed., 17, 1011 (1979).

5. N. A. J. M. van Aerle and P. J. Lemstra, Polym. J., 20, 131 (1988).

6. M. Yasuniwa, C. Nakafuku, and T. Takemura,
Polym. J., 4, 526 (1973).

7. D. C. Bassett and B. Turner, Nature (Phys. Sci.)., 240, 146 (1972).

8. D. C. Bassett, S. Block, and G. J. Piermarini, J. Appl. Phys., 45, 4146 (1974).

9. M. Yasuniwa, R. Enoshita, and T. Takemura, Jpn. J. Appl. Phys., 15, 1421 (1976).

10. K. Nagata, K. Tagashira, S. Taki, and T. Takemura, Jpn. J. Appl. Phys., 19, 985 (1980).

11. H. Tanaka and T. Takemura, Polym. J., 12, 355 (1980).

12. T. Yamamoto, H. Miyaji, and K. Asai, Jpn. J. Appl. Phys., 16, 1891 (1977).

13. N. Hiramatsu and S. Hirakawa, Polym. J., 12, 105 (1980).

14. G. Ungar and A. Keller, Polymer, 21, 1273 (1980).

15. A. S. Vaughan, G. Ungar, D. C. Bassett, and A. Keller, Polymer, 26, 726 (1985).

16. M. Yasuniwa, S. Tsubakihara, and C. Nakafuku, Polym. J., 20, 1075 (1988). 